Research Article

\title{
Prevalence and Associated Factors of Intestinal Parasitic Infections among Food Handlers in Mettu Town, Southwest Ethiopia
}

\author{
Solomon Yeshanew $\mathbb{D}^{1},{ }^{1}$ Melaku Tadege $\mathbb{D}^{2,},{ }^{2,3}$ and Abdulhakim Abamecha $\mathbb{D}^{4,5}$ \\ ${ }^{1}$ Department of Biology, Debre Markos University, P.O. Box 269, Debre Markos, Ethiopia \\ ${ }^{2}$ Department of Statistics, Bahir Dar University, Bahir Dar, P.O. Box 79, Amhara, Ethiopia \\ ${ }^{3}$ Department of Statistics, Injibara University, P.O. Box 40, Amhara, Ethiopia \\ ${ }^{4}$ Department of Biomedical Sciences, Mettu University, P.O. Box 318, Mettu, Ethiopia \\ ${ }^{5}$ Department of Medical Laboratory Sciences, Jimma University, P.O. Box 378, Jimma, Ethiopia
}

Correspondence should be addressed to Melaku Tadege; melakutadege@yahoo.com

Received 24 December 2020; Accepted 27 January 2021; Published 19 February 2021

Academic Editor: Pedro P. Chieffi

Copyright (c) 2021 Solomon Yeshanew et al. This is an open access article distributed under the Creative Commons Attribution License, which permits unrestricted use, distribution, and reproduction in any medium, provided the original work is properly cited.

\begin{abstract}
Food handlers are important sources of intestinal parasitic infection to the public and mass catering service areas. Several reports worldwide particularly in developing countries showed a high prevalence of intestinal parasitic infections among these groups of individuals. In an attempt to determine the prevalence of intestinal parasites, a cross-sectional study was carried out among food handlers in food establishment areas of Mettu town, Southwest Ethiopia. To collect sociodemographic characteristics of the study participants, a structured questionnaire and physical observation were employed. Stool specimens collected from each food handlers participated in the study were then examined using light microscopy of the wet mount followed by formol-ether concentration methods to see infection status. A total of 139 food handlers were enrolled in the study. Majority of them were females 89 (64\%), and 124 (89.2\%) of them had not taken any training related to food handling and preparation. Sixty-two (44.6\%) of the study participants were diagnosed with one or more intestinal parasites. The chi-square test showed that intestinal parasite infection was found associated with personal hygiene $(p \leq 0.0001)$, nail trimming status $(p \leq 0.0001)$, and hair cover status $(p \leq 0.040)$. The multivariable logistic regression indicated that the risk to be infected with intestinal parasites were related with older age $(p \leq 0.032)$, food handlers who had no hand wash practices $(p \leq 0.033)$, who had no food handling and preparation training ( $p \leq 0.005)$, poor personal hygiene $(p \leq 0.0001)$, who had not taken regular medical checkup $(p \leq 0.008)$, and whose nail was not trimmed $(p \leq 0.0001)$. The most abundant intestinal parasite identified was G. lamblia $(24,26.7 \%)$ followed by E. histolytica/dispar (22, 24.4\%), and A. lumbricoides (15, 16.7\%). Twenty-eight (45.2\%) study individuals were also found with mixed infections. The study thus revealed a high prevalence of intestinal parasitic infection among apparently healthy food handlers in food establishments of the study area. Mass drug administration for all food handlers working in food establishment areas of the town coupled with health education and training should be initiated urgently.
\end{abstract}

\section{Introduction}

Foodborne diseases continue to be a major global health problem and are the leading causes of morbidity and mortality in developing countries [1]. The World Health Organization (WHO) estimated $30 \%$ of the population in developed countries, suffers from foodborne diseases each year, whereas in developing countries, up to two million deaths are estimated per year, and more than $70 \%$ cases of diarrheal diseases are associated with the consumption of contaminated food [2].

Food handlers are an important source of contamination either as carriers of pathogens or through poor hygienic practices [3]. Subsequently, several reports showed a high 
prevalence of intestinal parasitic infections among food handlers in different parts of the world [4-7]. CDC also reported that approximately $20 \%$ of food-related infection is due to food handlers. A similar study in the USA also suggested that improper food handler practices contributed to approximately $97 \%$ of foodborne illnesses in food service establishment areas and homes [8].

In Ethiopia, the rate of intestinal parasitic infection among food handlers were conducted and ranged from 29 to $72 \%$ with different reports on the predominant species of parasites and hygiene practices [9-13]. The importance of food handlers as threats in the transmissions of intestinal parasitic infections has been due attention by several authors $[5,6,10,11]$. However, there is a scarcity of reported data on food handlers being identified as potential sources of intestinal parasitic infection from several parts of the country. According to the clinical data generated from Mettu Karl Hospital and a report from the Health Office of Mettu town, Southwestern Ethiopia, more than $50 \%$ of the population was estimated to be positive for intestinal parasites. Hence, an attempt is made in the present study to determine the prevalence of intestinal parasites and associated predisposing factors among food handlers serving in food establishment areas of the town.

\section{Methods}

2.1. Study Design and Area. A cross-sectional study was carried out among food handlers working in food establishment areas of Mettu town between March and June 2016. Mettu is a capital town of Mettu district and Ilu Aba Bora zone. The town is located approximately $600 \mathrm{~km}$ southwest of the capital Addis Ababa, Ethiopia. The district covers an area of $1452 \mathrm{~km}^{2}$ which lies between $8^{\circ} 6^{\prime}$ and $8^{\circ} 31^{\prime} \mathrm{N}$ latitude and $35^{\circ} 10^{\prime}$ and $35^{\circ} 50^{\prime} \mathrm{E}$ longitude plus an altitude range from 1000 to $2027 \mathrm{~m}$ above sea level. According to the 2013 national census, the number of inhabitants was estimated to be 54,792 (22,857 male and 31,935 female).

2.2. Study Population and Sample Size. Food handlers working at different kitchens of food service establishments and who were directly involved with food preparation and serving food to customers in the town were enrolled in the study. A total of 139 study subjects were selected randomly from 37 different cafeterias, hotels, butcher shops, restaurants, and mass catering serving establishments of the town. The sample size was calculated using single population proportion formula as described by Daniel (1999) [14] considering $95 \%$ confidence of interval, $5 \%$ margin of error, and prevalence rate of $44.1 \%$ of the previous studies [9].

2.3. Specimen Collection and Transport. Stool samples were collected from each food handler using clean stool cups by professional and trained medical laboratory technicians and transported into the Medical Laboratory Section of Mettu Karl hospital within half an hour.
2.4. Microscopic Examination of Stool. Stool specimen from all study participants were investigated using light microscopy of the direct wet mount followed by formol-ether concentration sedimentation methods according to the WHO guideline [15] in the hospital's parasitology research laboratory room.

2.5. Statistical Analysis. Analysis of the data was undertaken using SPSS version 20 software. Percentage and frequency distribution were used to determine the prevalence of intestinal parasitic infection. To see the association between the independent variables with parasitic infections status, the chisquare test was applied. Similarly, the effect of each exposure variables on the outcome variable was assessed using binary logistic regression analysis and $p$ value $<0.05$ was considered as a statistically significant level of intestinal parasitic infection.

\section{Results}

A total of 139 food handlers were selected randomly, and all of them agreed to participate in the study with a response rate of $100 \%$. Sixty-two $(44.6 \%)$ of the food handlers participated in the study were found to be positive for various types and species of intestinal parasites. Eighty-nine (64\%) of the participants were females, and 50 (36\%) were males. Majority of the study participants had less than a year of experience in food handling and preparation. Only 15 $(10.8 \%)$ of the food handlers enrolled in the study were certified in food preparation and handling training. Twentyfive $(18.0 \%)$ had medical checkup practice, and $79(56.8 \%)$ did not trim their fingernail. Among the 79 (56.8\%) fingernail nontrimmed study participants, 55 (88.7\%) were found positive with intestinal parasites. As such, the chisquare test approved that there was a statistically significant association between fingernail status and prevalence of intestinal parasitic infection $\left(X^{2}=46.352, p \leq 0.0001\right)$ at $5 \%$ significant levels. The statistics of the number of participants regard to have proper hand wash practice, and medical checkup were far from adequate. Concerning personal hygiene of the study participants, 27 (19.4\%), 61 (43.9\%), and $51(36.7 \%)$ were good, moderate, and poor status respectively. Around two-thirds (66.1\%) of intestinal parasitic infections were found among study subjects categorized under poor status. Regard to hair cover, 100 (71.9\%) of them had not used. Therefore the study deduced that intestinal parasite infections were highly associated with personal hygiene $(p \leq 0.0001)$, nail trimming status $(p \leq 0.0001)$, and hair cover status $(p \leq 0.040)$ (Table 1$)$.

Binary logistic regression analysis was conducted to determine major responsible factors for a high prevalence of intestinal parasitic infections in food handlers enrolled in the study. From univariate analysis, food handlers who had no hand wash practice, poor personal hygiene, and those who did not trim their nails were a predictor of intestinal parasitic infection. Since univariate analysis ignores the relationship between independent variables, we used multivariable analysis procedure for producing a valid conclusion about the population parameter. Therefore, multivariable logistic 
TABLE 1: Sociodemographic characteristics with respect to prevalence of intestinal parasites among food handlers in food establishment areas of Mettu town.

\begin{tabular}{|c|c|c|c|c|c|c|}
\hline \multirow{2}{*}{ Variables } & & \multirow{2}{*}{ Total examined $n(\%)$} & \multicolumn{2}{|c|}{ Infection status $n(\%)$} & \multirow{2}{*}{ Chi-square $\left(X^{2}\right)$} & \multirow{2}{*}{$p$ value } \\
\hline & & & Yes & No & & \\
\hline \multirow{3}{*}{ Gender } & Male & $50(36)$ & $19(30.6)$ & $31(40.3)$ & \multirow{3}{*}{1.378} & \multirow{3}{*}{0.240} \\
\hline & Female & $89(64)$ & $43(69.4)$ & $46(59.7)$ & & \\
\hline & Good & $27(19.4)$ & $3(4.8)$ & $24(31.2)$ & & \\
\hline \multirow[t]{2}{*}{ Personal hygiene } & Moderate & $61(43.9)$ & $18(29.0)$ & $43(55.8)$ & \multirow[t]{2}{*}{44.320} & \multirow[t]{2}{*}{0.0001} \\
\hline & Poor & $51(36.7)$ & $41(66.1)$ & $10(13.0)$ & & \\
\hline \multirow{2}{*}{ Hand wash habit } & Yes & $57(41)$ & $20(32.3)$ & $37(48.1)$ & \multirow{2}{*}{3.541} & \multirow{2}{*}{0.060} \\
\hline & No & $82(59)$ & $42(67.7)$ & $40(51.9)$ & & \\
\hline \multirow{2}{*}{ Training certified } & Yes & $15(10.8)$ & $6(9.7)$ & $9(11.7)$ & \multirow{2}{*}{0.144} & \multirow{2}{*}{0.704} \\
\hline & No & $124(89.2)$ & $56(90.3)$ & $68(88.3)$ & & \\
\hline \multirow{2}{*}{ Nail status } & Trimmed & $60(43.2)$ & $7(11.3)$ & $53(68.8)$ & \multirow{2}{*}{46.352} & \multirow{2}{*}{0.0001} \\
\hline & Not trimmed & $79(56.8)$ & $55(88.7)$ & $24(31.2)$ & & \\
\hline \multirow{2}{*}{ Medical checkup } & No & $114(82)$ & $55(88.7)$ & $59(76.6)$ & \multirow{2}{*}{3.401} & \multirow{2}{*}{0.065} \\
\hline & Yes & $25(18)$ & $7(11.3)$ & $18(23.4)$ & & \\
\hline \multirow{2}{*}{ Hair cover } & No & $100(71.9)$ & $50(80.6)$ & $50(64.9)$ & \multirow{2}{*}{4.199} & \multirow{2}{*}{0.040} \\
\hline & Yes & $39(28.1)$ & $12(19.4)$ & $27(35.1)$ & & \\
\hline \multirow{2}{*}{ Gowan cover } & No & $42(30.2)$ & $18(29.0)$ & $24(31.2)$ & \multirow{2}{*}{0.074} & \multirow{2}{*}{0.785} \\
\hline & Yes & $97(69.8)$ & $44(71.0)$ & $53(68.8)$ & & \\
\hline \multirow{4}{*}{ Work experience } & $<2$ years & $76(54.7)$ & $32(42.1)$ & $44(57.9)$ & \multirow{4}{*}{3.498} & \multirow{4}{*}{0.329} \\
\hline & $2-5$ years & $42(30.2)$ & $23(54.8)$ & $19(45.2)$ & & \\
\hline & $5-10$ years & $16(11.5)$ & $6(37.5)$ & $10(62.5)$ & & \\
\hline & $>10$ years & $5(3.6)$ & $1(20)$ & $4(80)$ & & \\
\hline
\end{tabular}

TABLE 2: Binary logistic regression analysis of intestinal parasitic infections and corresponding factors among food handlers at Mettu town food establishment areas.

\begin{tabular}{|c|c|c|c|c|c|}
\hline \multirow{2}{*}{ Variable } & \multirow{2}{*}{ Category } & \multicolumn{2}{|c|}{ Univariate analysis } & \multicolumn{2}{|c|}{ Multivariable analysis } \\
\hline & & COR $(95 \% \mathrm{CI})$ & $p$ value & AOR (95\% CI) & $p$ value \\
\hline Age & & $1.0110(0.9980,1.0240)$ & 0.1010 & $1.1760(1.0140,1.3640)$ & 0.0320 \\
\hline Hand wash habit & $\begin{array}{c}\text { Yes } \\
\text { No (ref) }\end{array}$ & $1.8500(1.0740,3.1870)$ & 0.0270 & $0.2220(0.0560,0.8830)$ & 0.0330 \\
\hline Training certified & $\begin{array}{c}\text { Yes } \\
\text { No (ref) }\end{array}$ & $1.5000(0.5340,4.2140)$ & 0.4420 & $0.0210(0.0010,0.3080)$ & 0.0050 \\
\hline Nail status & $\begin{array}{l}\text { Not trimmed } \\
\text { Trimmed (ref) }\end{array}$ & $7.5710(3.4420,16.6530)$ & 0.0001 & $10.4150(2.9380,18.9240)$ & 0.0001 \\
\hline Medical checkup & $\begin{array}{c}\text { Yes } \\
\text { No (ref) }\end{array}$ & $1.0730(0.7430,1.5490)$ & 0.7080 & $0.0410(0.0040,0.4340)$ & 0.0080 \\
\hline Gowan cover & $\begin{array}{c}\text { No } \\
\text { Yes (ref) }\end{array}$ & $1.3330(0.7240,2.4570)$ & 0.3560 & $2.9160(0.6740,12.6210)$ & 0.1520 \\
\hline Gender & $\begin{array}{c}\text { Male } \\
\text { Female (ref) }\end{array}$ & $1.6320(0.9220,2.8880)$ & 0.0930 & $0.8790(0.2330,3.3160)$ & 0.8480 \\
\hline
\end{tabular}

regression output stated that when the age of the food handlers increased by a year, the odds ratio of having intestinal parasitic infection were 1.176 , meaning the chance of infection with intestinal parasites were increased by $17.6 \%$ $(\mathrm{AOR}=1.1760$ (1.0140, 1.3640), $p \leq 0.0320)$. Food handlers who had a regular hand wash practice decreased the possibility to be infected by $0.778 \%(\mathrm{AOR}=0.2220(0.0560$, $0.8830), p \leq 0.0330)$. Likewise, study participants who acquired food handling and preparation training have decreased the chance of getting intestinal parasitic infections by $97.9 \% \quad(\mathrm{AOR}=0.0210 \quad(0.0010,0.3080), p \leq 0.0050)$. Similarly, participants of the study who had medical checkup decreased the probability of infection by $95.9 \%$ $(\mathrm{AOR} \leq 0.0410$ (0.0040, 0.4340), $p \leq 0.0080)$ (Table 2).
Sixty-two intestinal parasitic cases were diagnosed from study participants with the most frequently identified parasite G. lamblia which was found in $24(26.7 \%)$ food handlers followed by E. histolytica/dispar (22, 24.4\%) and A. lumbricoides $(15,16.7 \%)$. From the sixty-two positive food handlers for intestinal parasites, 46 (74.2\%) had protozoan and $44(71.0 \%)$ diagnosed with helminths. However, 28 (45.2\%) of them were found with mixed infections. Nine (14.5\%) were positive for E. histolytica/dispar and hookworm, and 6 (9.7\%) were by E. histolytica/dispar and A. lumbricoides. Similarly, G. lamblia with T. trichiura and $G$. lamblia with $A$. lumbricoides were also found concurrently with the prevalence of $8.2 \%$ in each case (Table 3). 
TABLE 3: Type and prevalence of intestinal parasites isolated from stool specimens of food handlers from food establishment areas of Mettu town.

\begin{tabular}{lcc}
\hline Intestinal parasite & Frequency & Percentage \\
\hline Protozoan & & \\
E. histolytica/dispar & 4 & 6.4 \\
G. lamblia & 14 & 22.6 \\
Helminths & & \\
A. lumbricoides & 4 & 6.4 \\
T. trichiura & 4 & 6.4 \\
H. nana & 2 & 3.2 \\
Hookworm & 3 & 4.8 \\
S. mansoni & 1 & 1.6 \\
Taenia spp. & 2 & 3.2 \\
Mixed infection & & \\
E. histolytica/dispar and A. lumbricoides & 6 & 9.7 \\
E. histolytica/dispar and Hookworm & 9 & 14.5 \\
E. histolytica/dispar and S. mansoni & 1 & 1.6 \\
E. histolytica/dispar and T. trichiura & 2 & 3.2 \\
G. lamblia and T. trichiura & 5 & 8.2 \\
G. lamblia and A. lumbricoides & 5 & 8.2 \\
Total & $\mathbf{6 2}$ & $\mathbf{1 0 0}$ \\
\hline
\end{tabular}

\section{Discussion}

Among food handlers working in food service establishment areas of Mettu town enrolled in the present study, nearly half (44.6\%) of them had been developing an intestinal parasitic infection. The finding was higher as compared with previous reports from southern Ethiopia (36\%) [16], Ghana (21\%) [6], Saudi Arabia (31.9\%) [17], and Kenya (24\%) [18]. However, it was in line with the report from Brazil (47.1\%) [19], Venezuela (48.7\%) [20], and Jordan (48.0\%) [21]. Similarly it was consistent with other investigations from elsewhere in Ethiopia such as Mekele (49.4\%) [10], Bahir Dar (41.1\%) [12], Yebu (44.1\%) [9], and Addis Ababa (45.3\%) [11]. In contrast to the present findings, reports from Jos, Nigeria (55.9\%) [22], from Hawassa, Ethiopia (63\%) [23], and Abeokuta, Nigeria (97\%) [24], were greater. This inconsistency might arise due to sample size and environmental variation, various habits of personal hygiene, and different settings of sociodemographic characteristics of the target population.

The study showed that intestinal parasitic infection was associated with nontrimmed fingernail status output, but other sociodemographic characteristics such as gender were not a predictor for infection. A similar study from Addis Ababa [11] was found in agreement with the present study. The study stated that hand wash habit, medical checking up status, and training were the most responsible factors of intestinal parasitic infection which was in line with reports from Yebu [9], Mekele [10], Nigeria [22], and southern Ethiopia [16]. The dominant parasite identified in the present study was G. lamblia 24 (26.7\%) followed by E. histolytica/dispar 22 (24.4\%). Fifteen (16.7\%) and 12 $(13.3 \%)$ of the study participants were found positive for A. lumbricoides and Hookworm infection, respectively. The report from Addis Ababa [11], southern Ethiopia [16], Bahirdar [12], and Mekele [10] showed that E. histolytical dispar and G. lamblia were the most abundant intestinal parasites diagnosed from study food handlers. However, a contrast finding from Yebu [9] and Gonder [25] reported A. lumbricoides and T. trichiura were detected at a high rate. This discrepancy could be due to the variation in the ecology and climatic differences of the study areas and the town level variations. Twenty-eight (20.1\%) food handlers enrolled in the study were diagnosed with mixed infection. Studies from Addis Ababa [11], Southern Ethiopia [16], Mekele [10], and Bahir Dar [12] similarly reported two or more intestinal parasites found concurrently on an individual.

\section{Conclusions}

Prevalence of intestinal parasites among food handlers in food service areas of Mettu town was $44.6 \%$. Poor handwashing habit, older age, nontrimmed fingernail, lack of proper training, and absence of regular medical checkup were the major predisposing factors of harbouring intestinal parasites among the study participants. We recommend an urgent need for mass drug administration and food preparation and handling training to all food handlers and managers of food service areas in the study locality.

\section{Abbreviations}

AOR: Adjusted odd ratio

CDC: Center for disease control

CI: Confident interval

COR: Crude odd ratio

SPSS: Statistical package for social sciences

USA: United States of America

WHO: World Health Organization.

\section{Data Availability}

The raw data used to support the findings of this study are available from the corresponding author upon request.

\section{Ethical Approval}

The study was carried out after receiving an ethical clearance endorsement from Research Ethical and Technical Review Clearance Committee (College of Medical Sciences, Mettu University, Ethiopia). Official permission was obtained from the health office of the town.

\section{Consent}

Verbal and written consents were also obtained from each study participants after informing them about the importance of the study.

\section{Disclosure}

Intestinal parasites positive study participants were treated free using standard drugs. 


\section{Conflicts of Interest}

The authors declare no conflicts of interest.

\section{Authors' Contributions}

This work was carried out in collaboration between all authors. SY and AA designed the study, performed the experiment, and wrote the protocol. SY and MT conducted the statistical analysis, managed the literature searches, and wrote the manuscript. All the authors read and approved the final version of the manuscript.

\section{Acknowledgments}

The authors would like to forward their gratitude to the Research and Technology Transfer Office of Mettu University for financial support of the study. Mettu Karl Hospital, Mettu Town Health Office, data collectors, and study participants shall also owe our heartfelt thanks for their cooperation. The study was supported financially by Mettu University. However, the University did not contribute to manuscript preparation, editing, approval, and publication processes.

\section{References}

[1] M. Jacob, Safe Food Handling: A Training Guide for Managers in Food Service Establishments, World Health Organization, Geneva, Switzerland, 1989.

[2] WHO, Food Safety and Foodborne Illness, World Health Organization, Geneva, Switzerland, 2007.

[3] F. K. Kaferstein, "Food safety the fourth pillar in the strategy to prevent infant diarrhea," Bulletin of the World Health Organization, vol. 81, no. 11, 2003.

[4] S. Khurana, N. Taneja, R. Thapar, M. Sharma, and N. Malla, "Intestinal bacterial and parasitic infections among food handlers in a tertiary care hospital of North India," Trop Gastroenterol, vol. 29, no. 4, pp. 207-209, 2010.

[5] M. Wakid, E. Azhar, and T. Zafar, "Intestinal parasitic infection among food handlers in the holy city of makkah during hajj Season1428 hegira (2007G)," Journal of King Abdulaziz University-Medical Sciences, vol. 16, no. 1, pp. 3952, 2009.

[6] P. F. Ayeh-Kumi, S. Quarcoo, G. Kwakye-Nuako, J. P. Kretchy, A. Osafo-Kantanka, and S. Mort, "Prevalence of intestinal parasitic infections among food vendors in accra, Ghana," The Journal of Tropical Medicine and Parasitology, vol. 32, pp. 1-8, 2009.

[7] H. A. Saeed and H. H. Hamid, "Bacteriological and parasitological assessment of food handlers in the omdurman area of Sudan," Journal of Microbiology, Immunology and Infection, vol. 43, no. 1, pp. 70-73, 2010.

[8] CDC, "Foodborne Illness," International Food Safety Consultancy, vol. 34, 2005.

[9] T. Tefera and G. Mebrie, "Prevalence and predictors of intestinal parasites among food handlers in Yebu town, southwest Ethiopia," PLoS One, vol. 9, no. 10, 2014.

[10] D. Nigusse and A. Kumie, "Food hygiene practices and prevalence of intestinal parasites among food handlers working in Mekelle university student's cafeteria, Mekelle," GARJSS, vol. 1, no. 4, pp. 65-71, 2012.
[11] A. Aklilu, D. Kahase, M. Dessalegn et al., "Prevalence of intestinal parasites, salmonella and shigella among apparently health food handlers of Addis Ababa University student's cafeteria, Addis Ababa, Ethiopia," BMC Research Notes, vol. 8, no. 1, p. 17, 2015.

[12] B. Abera, F. Biadegelgen, and B. Bezabih, "Prevalence of Salmonella typhi and intestinal parasites among food handlers in Bahir Dar Town, Northwest Ethiopia," The Ethiopian Journal of Health Development, vol. 24, no. 1, pp. 46-50, 2010.

[13] E. Tsega and F. Nadew, "The threat of ameobic cyst carriers among hospital food handlers," The Ethiopian Journal of Health Development, vol. 10, pp. 43-53, 1972.

[14] W. W. Daniel, Biostatistics: A Foundation for Analysis in the Health Sciences, 7th edition. John Wiley \& Sons, New York, NY, USA, 1999.

[15] WHO, Basic Laboratory Methods in Medical Parasitology. Parasitology Laboratory Manual, World Health Organization, Geneva, Switzerland, 1991.

[16] M. Mama and G. Alemu, "Prevalence and factors associated with intestinal parasitic infections among food handlers of Southern Ethiopia: cross sectional study," BMC Public Health, vol. 16, pp. 105-122, 2016.

[17] H. W. Majed, I. A. Esam, and A. Z. Tariq, "Intestinal parasitic infection among food handlers in the holy city of makkah during hajj season 1428 hegira (2007G) JKAU," Medical Sciences, vol. 16, no. 1, pp. 39-52, 2009.

[18] K. B. Gilbert, M. W. Paul, and M. N. Moses, "Prevalence of gastrointestinal parasitic infections among food handlers in eldoret municipality, Kenya," Journal of Biology Agriculture and Healthcare, vol. 4, no. 27, pp. 1-6, 2014.

[19] J. M. Costa-Cruz, M. L. G. Cardoso, and D. E. Marques, "Intestinal parasites in school food handlers in the city of Uberlândia, Minas Gerais, Brazil," Revista do Instituto de Medicina Tropical de São Paulo, vol. 37, no. 3, pp. 191-196, 1995.

[20] A. Freites, D. Colmenares, M. Pérez, M. García, and S. O. de Diaz, "Cryptosporidium spp infections and other intestinal parasites in food handlers from Zulia state, Venezuela," Venezuela Investigation Clinic, vol. 50, no. 1, pp. 13-21, 2009.

[21] A. B. Al-Lahham, M. Abu-Saud, and A. A. Shehabi, "Prevalence of Salmonella, Shigella and intestinal parasites in food handlers in Irbid, Jordan," Journal of Diarrhoeal Diseases Research, vol. 8, no. 4, pp. 160-162, 1990.

[22] J. G. Damen, E. U. Cosmas, and C. A. Damak, "Intestinal parasitosis among food handlers in Jos, north Central Nigeria," Danish Journal of Agriculture and Animal Sciences, vol. 34, pp. 53-58, 2015.

[23] S. Teklemarium, B. Roma, S. Sorsa, S. Worku, and L. Erosie, "Assessment of sanitaryand hygienic status of catering establishments of Awassa town," Danish Journal of Agriculture and Animal Sciences, vol. 14, no. 1, pp. 91-98, 2000.

[24] O. A. Idowu and S. A. Rowland, "Oral fecal parasites and personal hygiene of food handlers in Abeokuta, Nigeria," African Health Sciences, vol. 6, no. 3, pp. 160-164, 2006.

[25] G. Andargie, A. Kassu, F. Moges, M. Tiruneh, and K. Huruy, "Prevalence of bacteria and intestinal parasites among foodhandlers in gondar town, northwest Ethiopia," Journal of Health, Population, and Nutrition, vol. 26, no. 4, pp. 451-455, 2008. 